\title{
ABSORPTION RECOVERY OF THE PYRIMIDOCARBOCYANINE DYE PYC
}

\author{
A. PENZKOFER and F. OSSIG \\ Naturwissenschaftliche Fakultät II - Physik, Universität Regensburg, D-8400 Regensburg, Federal Republic of Germany
}

Received 22 August 1988; in final form 14 October 1988

\begin{abstract}
The ground-state absorption recovery time of the dye 1,3,1',3'-tetramethyl-2,2' -dioxopyrimido-6,6'-carbocyanine hydrogen sulfate (PYC) dissolved in hexafluoroisopropanol, determined by picosecond pump and time-delayed probe pulse transmission measurements, is found to be $14 \pm 2 \mathrm{ps}$. The $\mathrm{S}_{1}-\mathrm{S}_{n}$ excited-state absorption cross-section at $527 \mathrm{~nm}$ is determined by pump pulse bleaching experiments and found to be $\sigma_{\mathrm{ex}}=(3 \pm 0.7) \times 10^{-17} \mathrm{~cm}^{2}$.
\end{abstract}

\section{Introduction}

The dye 1,3,1', 3' -tetramethyl-2,2'-dioxopyrimido-6,6'-carbocyanine hydrogen sulfate (PYC) dissolved in hexafluoroisopropanol (HFIP) [1,2] was applied recently to efficient phase-matched thirdharmonic generation $[3,4]$ and to two-photon pumped travelling-wave laser action [5]. Picosecond pump pulses of a passively mode-locked $\mathrm{Nd}$ : glass laser were used in these experiments. Fluorescence quantum yield measurements indicate a fluorescence lifetime of $\tau_{\mathrm{F}} \approx 13 \pm 2$ ps [1].

In this paper the saturable absorption behaviour of PYC dissolved in HFIP is studied. The groundstate absorption recovery time $\tau_{\mathrm{R}}$ and the excitedstate absorption cross-section $\sigma_{\mathrm{ex}}$ at the pump laser frequency $\left(\lambda_{\mathrm{P}}=527 \mathrm{~nm}\right)$ are determined. The absorption recovery time is found to be equal to the fluorescence lifetime. The weak excited-state absorption makes the dye suitable for fast mode-locking applications.

\section{Experimental}

The experimental apparatus for the excited-state absorption cross-section determination is shown in fig. 1a. A passively mode-locked Nd: phosphate glass laser is used. A single pulse is selected and amplified. The second harmonic is generated in a KDP crystal (pulse duration $\Delta t_{\mathrm{P}} \approx 4 \mathrm{ps}$ ). The peak intensity of the pump pulses at the dye sample is determined by twophoton transmission measurements through a rutile crystal [6] (photodetectors PD1 and PD2). The en-

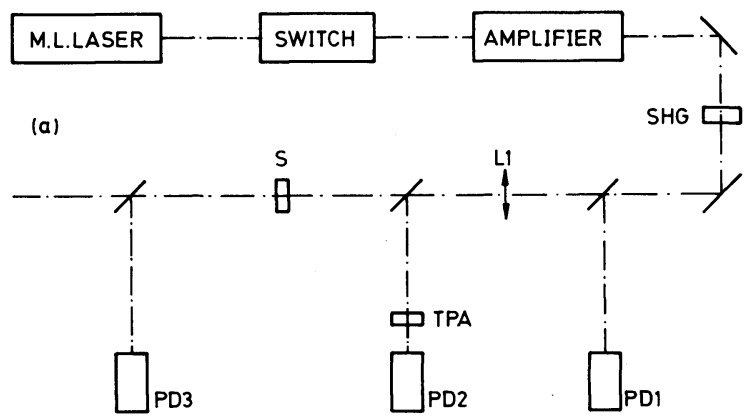

(b)

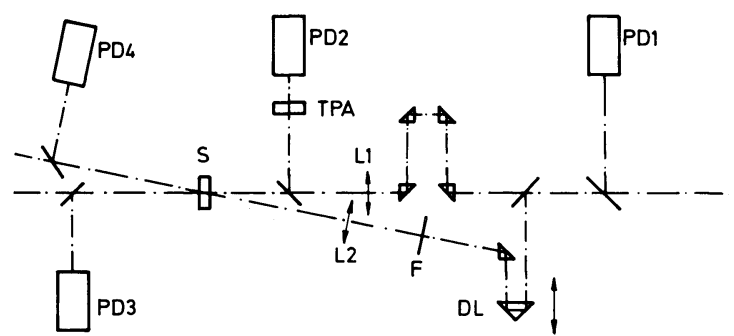

Fig. 1. Experimental apparatus. (a) Arrangement for bleaching measurements (determination of excited-state absorption crosssection). (b) Pump and probe arrangement for absorption recovery time measurement. SHG, KDP crystal for second-harmonic generation. L1, L2, lenses. S, dye sample. PD1-PD4, photodetectors. TPA, rutile crystal for peak intensity detection [6]. DL, optical delay line. F, filter. 
ergy transmission through the dye sample is recorded with the detectors PD1 and PD3.

The ground-state absorption recovery time is measured using the experimental arrangement shown in fig. $1 \mathrm{~b}$. A probe pulse is split off from the pump pulse, sent through a variable optical delay line, and passed through the dye sample region excited by the pump pulse. The input pump pulse peak intensity is measured as above. The pump pulse transmission (detectors PD1 and PD3) and the probe pulse transmission (detectors PD1 and PD4) through the sample are detected.

\section{Results}

The absorption and emission cross-section spectrum of PYC dissolved in HFIP is shown in fig. 2 [1]. The pump laser wavelength $\lambda_{P}$ and excited-state

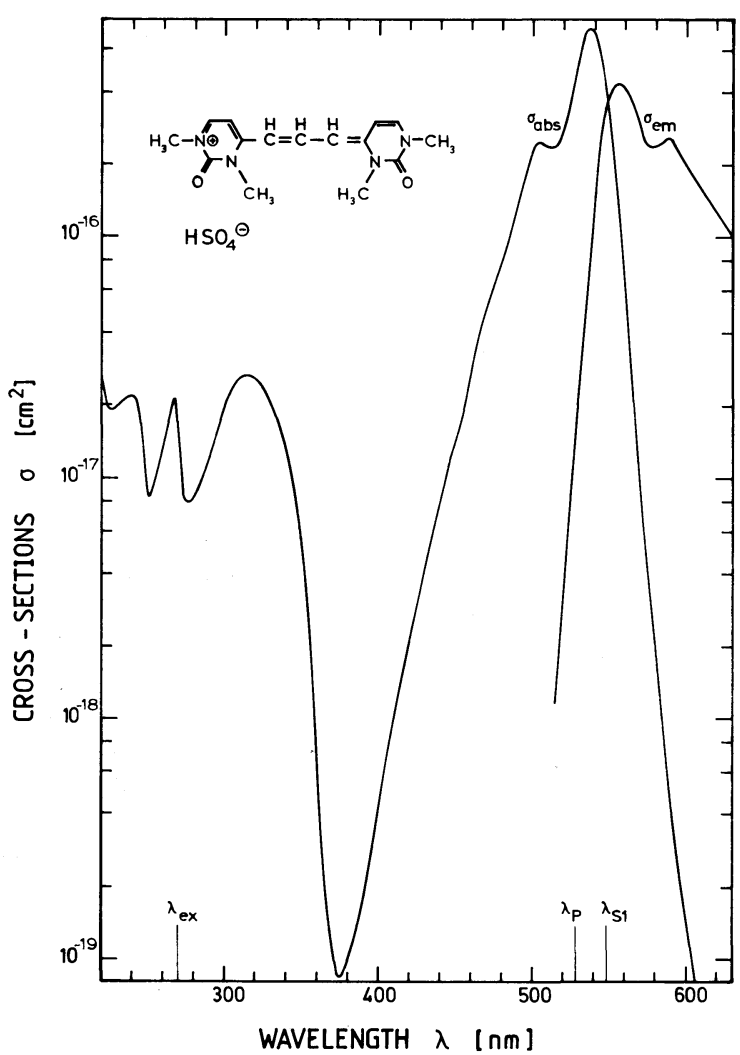

Fig. 2. Absorption and emission cross-section spectrum of PYC in hexafluoroisopropanol (from ref. [1]). absorption wavelength $\lambda_{\mathrm{ex}}$ are indicated $\left(\lambda_{\mathrm{ex}}^{-1}=\right.$ $\lambda_{\mathrm{P}}^{-1}+\lambda_{\mathrm{S} 1}^{-1} ; \lambda_{\mathrm{S} 1}$ is the electronic $\mathrm{S}_{0}-\mathrm{S}_{1}$ transition wavelength). The structural formula of PYC is included in fig. 2.

The determination of the ground-state absorption recovery time [7-9] and of the excited-state absorption cross-section [10] are discussed separately in the following.

\subsection{Ground-state absorption recovery time}

After pump pulse excitation the $S_{1}$-level population $N \mathrm{~S}_{1}^{\mathrm{pu}}$ relaxes according to

$N \mathrm{Su}_{1}^{\mathrm{pu}}(t)=N_{S 1,0}^{\mathrm{pu}} f(t)$,

where $f(t)$ is the $\mathrm{S}_{1}$-state relaxation function. $N \mathrm{~S}_{1}^{\mathrm{pu}}(t)$ is determined by the probe pulse transmission measurement. The probe pulse transmission $T_{\mathrm{pr}}$ is

$$
\begin{aligned}
& T_{\mathrm{pr}}(t)=\exp \left\{-\sigma_{\mathrm{P}} l\left[N_{0}-N_{\mathrm{S} 1}^{\mathrm{pu}}(t)-N_{\mathrm{S} 1}^{\mathrm{rr}}\right]\right. \\
& \left.-\sigma_{\mathrm{ex}} l\left[N_{\mathrm{S} 1}^{\mathrm{pu}}(t)+N_{\mathrm{S} 1}^{\mathrm{pr}}\right]\right\} .
\end{aligned}
$$

$N \mathrm{Sr}_{1}^{\mathrm{pr}}$ is the $\mathrm{S}_{1}$-state population caused by the probe pulse. $N_{0}$ is the total number density of dye molecules. $l$ is the sample length and $\sigma_{\mathrm{P}}$ is the ground-state absorption cross-section at the pump pulse laser frequency. $T_{0}=\exp \left(-\sigma_{\mathrm{L}} N_{0} l\right)$ is the small-signal transmission at $\lambda_{\mathrm{P}}$.

Solving eq. (2) for $N_{\mathrm{S}_{1}}^{\mathrm{pu}}(t)$ gives

$N \mathrm{pu}_{1}^{\mathrm{u}}(t)=\frac{1}{\left(\sigma_{\mathrm{P}}-\sigma_{\mathrm{ex}}\right) l} \ln \left(\frac{T_{\mathrm{pr}}(t)}{T_{0}}\right)-N_{\mathrm{S} 1}^{\mathrm{pr}}$.

$N \mathrm{Sr}_{1}^{\mathrm{pr}}$ is given by

$N \mathrm{p}_{1}^{\mathrm{r}}=\frac{1}{\left(\sigma_{\mathrm{P}}-\sigma_{\mathrm{ex}}\right) l} \ln \left(\frac{T_{\mathrm{pr}}(\infty)}{T_{0}}\right)$.

Insertion of eq. (4) into eq. (3) gives

$N$ pi $(t)=\frac{1}{\left(\sigma_{\mathrm{P}}-\sigma_{\mathrm{ex}}\right) l} \ln \left(\frac{T_{\mathrm{pr}}(t)}{T_{\mathrm{pr}}(\infty)}\right)$.

The temporal dependence of $\ln \left[T_{\mathrm{pr}}(t) / T_{\mathrm{pr}}(\infty)\right]$ at a fixed pump pulse peak intensity determines the $S_{1}$ state relaxation function $f(t)$ (eq. (1)). 
If the pump pulse intensity varies from shot to shot the relaxation function $f(t)$ may be approximately recorded by normalizing the transmission measurements according to

$f(t) \approx \frac{\ln \left[T_{\mathrm{pr}}(t) / T_{\mathrm{pr}}(\infty)\right]}{\ln \left(T_{\mathrm{pu}} / T_{0}\right)}=f_{\mathrm{m}}(t)$

$T_{\mathrm{pu}}$ is the pump pulse transmission (measured with photodetectors PD1 and PD3 in fig. 1b).

The $f_{\mathrm{m}}(t)$ function determined experimentally is plotted in fig. 3. It shows single-exponential decay with an absorption recovery time constant of $\tau_{\mathrm{R}}=14 \pm 2$ ps.

The measured absorption recovery time is - within the error bars - equal to the fluorescence lifetime [1]. This equality indicates that the $S_{1}$-state relaxes to the $\mathrm{S}_{0}$ ground state without a long-lived intermediate state (no remarkable intersystem crossing; fast relaxation due to internal conversion or twisted internal charge transfer [11]).

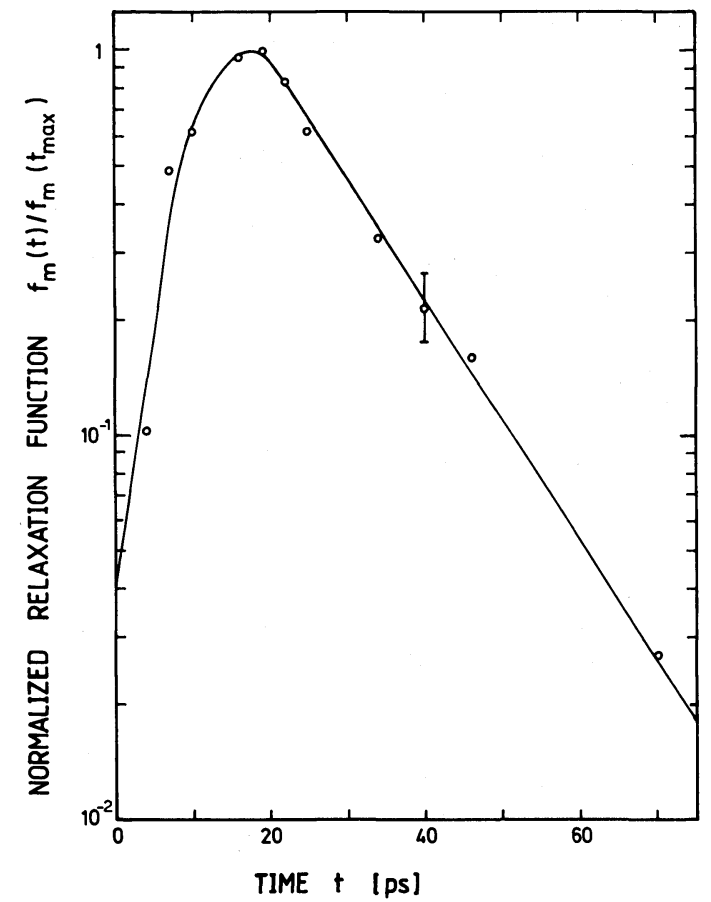

Fig. 3. Normalized time-delayed probe pulse transmission for absorption recovery time determination (eq. (6)). Single exponential decay with $\tau_{\mathrm{R}}=14 \mathrm{ps}$ is observed.

\subsection{Excited-state absorption cross-section}

The excited-state absorption cross-section of PYC in HFIP at the pump laser frequency is determined by measuring the energy transmission versus pump pulse peak intensity and comparing with calculations. The measured energy transmission points are plotted in fig. 4 (dye concentration $10^{-4} \mathrm{~mol} / \mathrm{dm}^{3}$, sample length $l=0.1 \mathrm{~cm})$. An estimate of the excited-state absorption cross-section is obtained by assuming complete ground-state depletion at the highest pump pulse intensity applied and setting the highest transmission, $T_{\max }$, equal to the $\mathrm{S}_{1}-\mathrm{S}_{n}$ excited-state transmission, i.e.

$T_{\max } \approx \exp \left(-\sigma_{\mathrm{ex}} N_{0} l\right)$.

Application of eq. (7) gives $\sigma_{\mathrm{ex}} \approx 3.2 \times 10^{-17} \mathrm{~cm}^{2}$.

A more rigorous determination of $\sigma_{\mathrm{ex}}$ using a fourlevel energy diagram (inset of fig. 4 ) is described in ref. [14]. The solid curve in fig. 4 is calculated with

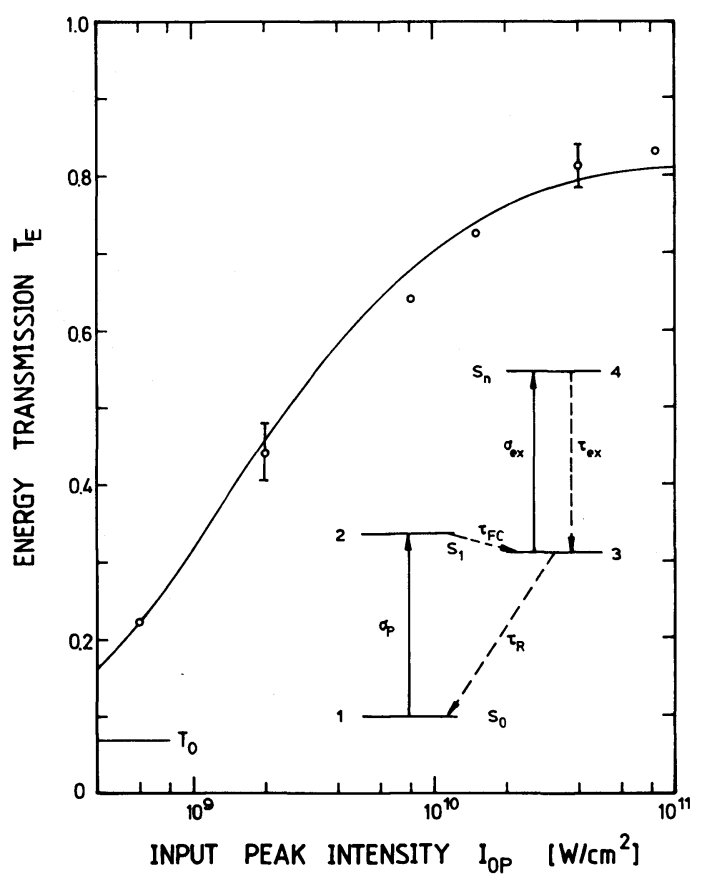

Fig. 4. Energy transmission, $T_{\mathrm{E}}$, versus input pump pulse peak intensity, $I_{0 \mathrm{P}} .10^{-4}$ molar PYC in HFIP. Sample length $l=1 \mathrm{~mm}$. Curve is calculated for $\sigma_{\mathrm{ex}}=3 \times 10^{-17} \mathrm{~cm}^{2}$ and $\sigma_{\mathrm{P}}=4.4 \times 10^{-16}$ $\mathrm{cm}^{2}, \tau_{\mathrm{R}}=14 \mathrm{ps}, \tau_{\mathrm{FC}}=0.7 \mathrm{ps}, \tau_{\mathrm{ex}}=0.1 \mathrm{ps}[12], \tau_{\mathrm{or}}=150 \mathrm{ps}$ (reorientation time [13]). Inset: Energy level diagram for saturable absorption calculations [14]. 
the rate equation system of ref. [14] for $\sigma_{\mathrm{ex}}=3$ $\times 10^{-17} \mathrm{~cm}^{2}$. The ratio of excited-state absorption cross-section, $\sigma_{\mathrm{ex}}$, to the ground-state absorption cross-section, $\sigma_{\mathrm{P}}$, is $\sigma_{\mathrm{ex}} / \sigma_{\mathrm{P}} \approx 0.07$ indicating a high bleaching capability. The dye PYC dissolved in HFIP is thought to be a good fast saturable absorber [15] in the spectral region between 480 and $580 \mathrm{~nm}$ [16].

\section{Conclusions}

The described analyses indicate that PYC dissolved in HFIP is a fast saturable absorber $\left(\tau_{R} \approx 14\right.$ ps) with low excited state absorption $\left(\sigma_{\mathrm{ex}}=(3 \pm 0.7) \times 10^{-17} \mathrm{~cm}^{2}\right.$ at $\lambda_{\mathrm{P}}=527 \mathrm{~nm} ; \sigma_{\mathrm{ex}} / \sigma_{\mathrm{P}}$ $\approx 0.07)$. The dye should be applicable as a saturable absorber for second harmonic light pulses of modelocked Nd lasers and as a fast mode-locking dye for passive or hybrid mode-locking of both pulsed and cw mode-locked dye lasers in the spectral region between 480 and $580 \mathrm{~nm}$.

\section{Acknowledgement}

The authors are indebted to Professor K.H. Drexhage, Universität Siegen, and Dr. U. Mayer and Dr. G. Wagenblast, BASF Ludwigshafen, for providing the dye PYC. They thank the Deutsche Forschungsgemeinschaft for financial support and the
Rechenzentrum of the University for the allocation of computer time.

\section{References}

[1] A. Penzkofer, W. Leupacher, B. Meier, B. Runde and K.H. Drexhage, Chem. Phys. 115 (1987) 143.

[2] A. Penzkofer and W. Leupacher, Opt. Quant. Electron. 19 (1987) 327.

[3] W. Leupacher, A. Penzkofer, B. Runde and K.H. Drexhage, Appl. Phys. B 44 (1987) 133.

[4] A. Penzkofer and W. Leupacher, Opt. Quant. Electron. 20 (1988) 277

[5] P. Qiu and A. Penzkofer, Appl. Phys. B, to be published.

[6] A. Penzkofer and W. Falkenstein, Opt. Commun. 16 (1976) 247.

[7] R.I. Scarlet, J.F. Figueria and H. Mahr, Appl. Phys. Letters 13 (1968) 71.

[8] S.L. Shapiro, ed., Topics in applied physics, Vol. 18. Ultrashort light pulses (Springer, Berlin, 1977).

[9] W. Kaiser, ed., Topics in applied physics, Vol. 60. Ultrashort laser pulses and applications (Springer, Berlin, 1988).

[10] A. Penzkofer and W. Blau, Opt. Quant. Electron. 15 (1983) 325.

[11] W. Rettig, Angew. Chem. Intern. Ed. Engl. 25 (1986) 971.

[12] A. Penzkofer and W. Falkenstein, Opt. Quant. Electron. 10 (1978) 399.

[13] H.E. Lessing and A. von Jena, in: Laser handbook, Vol. 3, ed. M.L. Stitch (North-Holland, Amsterdam, 1979) ch. B6, p. 753.

[14] G. Grönninger and A. Penzkofer, Opt. Quant. Electron. 16 (1984) 225.

[15] A. Penzkofer, Appl. Phys. B 46 (1988) 43.

[16] W. Blau, W. Dankesreiter and A. Penzkofer, Chem. Phys. 85 (1984) 473. 